\title{
USO Y ABUSO DE LOS AUTOINFORMES EN LA EVALUACIÓN DE LOS TRASTORNOS DE PERSONALIDAD
}

\author{
JAVIER FERNÁNDEZ-MONTALVO ${ }^{\prime}$ Y ENRIQUE ECHEBURÚA ${ }^{2}$ \\ 'Universidad Pública de Navarra, Pamplona \\ ${ }^{2}$ Facultad de Psicología, Universidad del País Vasco, San Sebastián
}

\begin{abstract}
Resumen: En este artículo se lleva a cabo una revisión de las discrepancias observadas en los estudios de comorbilidad entre los trastornos mentales del Eje I y los trastornos de personalidad. Las investigaciones llevadas a cabo hasta la fecha muestran un amplio rango en la tasa de comorbilidad, con variaciones en función del tipo de instrumento de evaluación utilizado. Los estudios que recurren a cuestionarios muestran tasas significativamente más altas de trastornos de personalidad que los llevados a cabo con entrevistas clínicas. Los autoinformes presentan, por tanto, una tendencia a sobrediagnosticar trastornos de personalidad. Las entrevistas clínicas, en cambio, son más estrictas y conservadoras, por lo que las tasas de prevalencia son más bajas y ajustadas, incluso con diferencias significativas cuando se utilizan ambos tipos de instrumentos con una misma muestra clínica. Se ejemplifican estos datos con estudios concretos llevados a cabo en el ámbito del alcoholismo, la ludopatía, la adicción a la cocaina y los trastornos de la conducta alimentaria. Por último, se comentan las implicaciones de este hecho para la práctica clínica cotidiana y para la investigación futura.
\end{abstract}

Palabras clave: Trastornos de personalidad, autoinformes, entrevistas clínicas, evaluación.

\section{Use and abuse of self-reports in the assessment of personality disonders}

\begin{abstract}
In this paper an analysis of the observed discrepancies in studies about the comorbidity between mental disorders of Axis I and personality disorders was carried out. Research shows a wide range of the comorbidity rates, with variations in function of the kind of assessment tool used. The studies that use self-reports show significantly higher rates of personality disorders than the ones using clinical interviews. Therefore, self-reports have a tendency to over-diagnose personality disorders. However, clinical interviews are stricter and more conservative and so the prevalence rates are lower, even with significant differences when both types of assessment tools are used with the same clinical sample. These data are illustrated with specific studies carried out in some clinical disorders: alcoholism, pathological gambling, cocaine dependence and eating disorders. Lastly, implications of this study for clinical practice and future research are commented upon.
\end{abstract}

Key words: Personality disorders, self-reports, clinical interviews, assessment.

\section{INTRODUCCIÓN}

En los últimos años se ha producido un interés creciente por el estudio de la comorbilidad entre los diferentes trastornos mentales. Este hecho se relaciona probablemente con la dificultad cada vez mayor de encontrar en consulta casos clínicos puros, aquejados de un solo trastorno psicopatológico y sin comorbilidad

Recibido: 15 de diciembre de 2004. Aceptado: 6 de mayo de 2005.

Correspondencia: Javie Fernández-Montalvo, Departamento de Psicología y Pedagogia. Universidad Pública de Navarra, Campus de Arrosadía, 31006 Pamplona. Correo-e: fernandez.montalvo@unavarra.es. asociada. De hecho, la comorbilidad entre trastornos empieza a ser más la regla que la excepción (Fernández-Montalvo, Landa y López-Goñi, 2005). En este sentido destaca enormemente la comorbilidad de los distintos cuadros clínicos del Eje I con los trastornos de la personalidad. Ello implica una necesidad de mejorar los modelos explicativos de este hecho concreto, así como de depurar los instrumentos de evaluación existentes o de crear otros nuevos, para poder ofrecer, en último término, una respuesta terapéutica adecuada a los pacientes. Lo cierto es que, al margen de la necesidad de depurar la categoría actual de los trastornos de 
Javier Fernández-Montalvo y Enrique Echeburúa

la personalidad, hoy en día no se conocen con precisión las vías de relación entre la personalidad y la psicopatología (De la Gándara y Medina, 1998).

Desde una perspectiva clínica es fundamental llegar a determinar el alcance de los trastornos de personalidad en el cuadro clínico presentado por el paciente. En primer lugar, existe cierta evidencia empírica de la influencia que los trastornos de personalidad tienen en el pronóstico terapéutico del caso clínico (Cangas y Olivencia, 2001; Nace, Davis y Gaspari, 1991). En segundo lugar, la comorbilidad de un trastorno mental con algún trastorno de personalidad aumenta considerablemente las dificultades en el manejo clínico de estos pacientes (Cangas y Olivencia, 2001; Pedrero y Segura, 2003). En tercer lugar, en el ámbito específico de las adicciones, algunos estudios han encontrado una relación significativa entre la presencia de un trastorno de personalidad y el mayor riesgo de recaída o abandono (Fernández-Montalvo et al., 2004; Verheul, Van den Brink y Hartgers, 1998a). Y, por último, también en el ámbito de las adicciones, se ha llegado a señalar la existencia de una eficacia terapéutica diferencial en función del tipo concreto de trastorno de personalidad presente (Poldrugo y Forti, 1988).

No hay más que echar un vistazo a las publicaciones recientes para percatarse de que en las dos últimas décadas se ha producido un desarrollo espectacular de los instrumentos de medida en el ámbito de los trastornos de personalidad (Cañas de Paz, 1998; Samino y Lorenzo, 2003). Los cuestionarios autoadministrados y las entrevistas estructuradas son los sistemas de medida más utilizados. Si bien hay un cierto acuerdo sobre la superioridad de las entrevistas sobre los cuestionarios en este campo, las entrevistas requieren un tiempo de administración prolongado y un personal especializado. Por ello, los cuestionarios son los instrumentos a los que más se recurre en la clínica y en la investigación.

El objetivo de este artículo es hacer una valoración específica de los autoinformes en comparación con las entrevistas en este ámbi- to, así como analizar las discrepancias observadas en los estudios de comorbilidad entre los cuadros clínicos del Eje I y los trastornos de personalidad.

\section{EVALUACIÓN DE LOS TRASTORNOS DE PERSONALIDAD: ¿ENTREVISTAS O CUESTIONARIOS?}

La evaluación de los trastornos mentales en general se ha realizado con diferentes sistemas de medida (Echeburúa, Amor y Corral, 2003): entrevistas (más o menos estructuradas), autoinformes (cuestionarios, inventarios y escalas), pruebas de observación y registros psicofisiológicos. Muchas de estas medidas correlacionan altamente entre sí y no aportan una información adicional significativa, excepto que contribuyen a aumentar la fiabilidad de la evaluación. De todas ellas, los autoinfomes, desde una perspectiva de costes y beneficios, son, en principio, preferibles a las pruebas de observación (más costosas y dificiles de organizar) y a los registros psicofisiológicos, que presentan muchos problemas metodológicos.

Sin embargo, los autoinformes utilizados en la evaluación psicopatológica son muy vulnerables a la manipulación, que reviste, habitualmente, la forma de una simulación de los síntomas, de una exageración (sobresimulación) de sintomas leves 0 , a veces, de un ocultamiento (disimulación) de los síntomas (Calcedo, 2000; Echeburúa et al., 2003). Estas limitaciones se acentúan más aún cuando se trata de evaluar los trastornos de personalidad. Debido al carácter egosintónico de la mayor parte de ellos, se producen con frecuencia numerosos sesgos o errores en las respuestas de los sujetos. La falta de reconocimiento del problema, la escasa motivación, la simulación o la deseabilidad social son factores que se acentúan en este tipo de alteraciones. Por ello, en general, el valor de los autoinformes es más limitado aún en el ámbito de los trastornos de personalidad (Echeburúa et al., 2003; Widiger y Frances, 1987). 
De ahí que, según algunos autores (Dutton, 2003), es mejor no recurrir a los cuestionarios autoadministrados para evaluar los trastornos de personalidad. Incluso en algunos campos - la evaluación de los maltratadores a la pareja, por ejemplo - hay una polémica abierta en cuanto a la fiabilidad y validez de los resultados obtenidos en los estudios que utilizan autoinformes para detectar los trastornos de personalidad (Dutton, 2003; Gondolf, 2003).

En contraste con estas limitaciones, las entrevistas clínicas son de mayor utilidad para la evaluación de los trastornos de personalidad. Las entrevistas, sobre todo en el ámbito de este tipo de cuadros clínicos, ofrecen un rendimiento muy superior a los autoinformes. Así, por ejemplo, permiten evaluar aspectos de difícil valoración con escalas autoaplicadas: conciencia de enfermedad, ideas delirantes, etcétera. Además, ofrecen al clínico la oportunidad de preguntar al paciente ejemplos concretos de situaciones reales, que, entre otras cosas, sirven para distinguir los problemas situacionales de los rasgos de personalidad.

No obstante, las entrevistas también están sujetas a fuentes de distorsión. Como señalan algunos autores (Echeburúa et al., 2003), las entrevistas, a diferencia de los autoinformes, carecen habitualmente de baremos estandarizados. Asimismo los entrevistadores pueden dar origen a diferentes errores: anotar síntomas inexistentes, no percatarse de indicios no verbales clínicamente significativos, inferir la gravedad de un síntoma a partir de lo que se evalúa en otro, etcétera. Estos errores son más acentuados cuando los entrevistadores cuentan con poca formación o experiencia clínica, no tienen un equilibrio emocional adecuado o son muy rígidos en los modelos teóricos adoptados. En cualquier caso, y a pesar de los sesgos señalados, no se debe olvidar que no es posible llevar a cabo un diagnóstico clínico únicamente a partir de los resultados obtenidos en un cuestionario autoadministrado. El diagnóstico psicopatológico requiere siempre de un juicio clínico por parte de un terapeuta, y ello sólo es posible mediante la utilización de entrevistas heteroaplicadas.
Sin embargo, las entrevistas estructuradas existentes en la actualidad para la evaluación de los trastornos de personalidad -el Structured Clinical Interview for DSM-III (SCID-II) (Spitzer, Williams y Gibbon, 1989) o el International Personality Disorder Examination (IPDE) (Loranger, 1995), por citar las más utilizadasson muy largas y farragosas $y$, por tanto, requieren mucho tiempo para su aplicación. Ello las convierte en poco operativas, sobre todo en Centros de Salud Mental, muy marcados por la presión asistencial existente. Como consecuencia, se tiende a prescindir de ellas y a recurrir, en aquellos casos en los que se sospecha la presencia de un trastorno de personalidad, a los cuestionarios -principalmente el MCMI-II (Millon, 1997)—, que son más cómodos para el clínico y no requieren un tiempo tan elevado para su cumplimentación. Ahora bien, la pregunta resultante es la siguiente: ¿son fiables los resultados obtenidos con este tipo de instrumentos a la hora de evaluar los trastornos de personalidad?

\section{DISCREPANCIA ENTRE LOS CUESTIONARIOS Y LAS ENTREVISTAS}

Los estudios llevados a cabo hasta la fecha sobre la comorbilidad de los cuadros clínicos del Eje I con los trastornos de personalidad muestran unos resultados muy heterogéneos. Ello se debe, probablemente, a que no existe una validación consistente de las categorías diagnósticas de los trastornos de personalidad, lo que pone en duda su fiabilidad diagnóstica e incluso su validez como constructo teórico (Baca-Baldomero, 1999; Girolamo y Reich, 1993). De hecho, los trastornos de personalidad constituyen uno de los puntos más conflictivos de la psicopatología. Asimismo los intentos de evaluación sistemática de estos trastornos son muy recientes (Bernardo y Roca, 1999; Caba1lo, 2004).

No obstante, y al margen de las imprecisiones conceptuales, resulta curioso observar las amplias discrepancias entre los distintos estudios, en función de los instrumentos de evalua- 
ción utilizados para detectar los trastornos de personalidad. El grado de concordancia entre los autoinformes y las entrevistas clínicas es muy bajo, lo que indica una baja fiabilidad en el diagnóstico de estos trastornos. Todo ello indica la necesidad de interpretar con precaución los datos obtenidos con cuestionarios autoadministrados, que están muy condicionados por la subjetividad del paciente (Hyler et al., 1989; Zimmerman y Coryell, 1990). El tipo de instrumento de evaluación utilizado resulta, por tanto, fundamental a la hora de valorar la presencia de un trastorno de personalidad en el ámbito clínico.

Es más, según el estudio de revisión de Perry (1992), que confirma la existencia de un bajo grado de concordancia entre los distintos instrumentos de evaluación (Kappa $=0,25$ ), hay una mayor concordancia entre diferentes entrevistas estructuradas que entre éstas y los cuestionarios autoadministrados.

En los siguientes párrafos se presentan, a modo de ejemplo, algunas de estas discrepancias observadas en diversos estudios y referidas a diferentes cuadros clínicos. No se trata, en modo alguno, de llevar a cabo una revisión exhaustiva de todos los estudios sobre comorbilidad con los trastornos de personalidad, sino de ejemplificar, en algunos trastornos psicopatológicos, este tipo de discrepancias observadas según sean los instrumentos de evaluación utilizados.

\section{ALCOHOLISMO}

Por lo que se refiere específicamente al alcoholismo - uno de los cuadros clínicos en los que más se ha estudiado la comorbilidad con los trastornos de personalidad-, las tasas de prevalencia encontradas oscilan entre el $24 \%$ y el $80 \%$. A su vez, los trastornos de personalidad más frecuentemente hallados son también muy variables, pero destacan, entre ellos, el antisocial y el límite, dentro del grupo B, y el trastorno de la personalidad por evitación, dentro del grupo $\mathrm{C}$ (Fernández-Montalvo y Landa, 2003).
Esta amplia variabilidad de resultados se ha atribuido a diferentes factores (Verheul, Van den Brink y Hartgers, 1995): los instrumentos de evaluación utilizados, el contexto del estudio (pacientes internos o ambulatorios), la distribución por sexos de la muestra, los criterios de inclusión o exclusión aplicados (por ejemplo, la existencia de consumo añadido de otras drogas), el sistema de clasificación empleado y el momento de la evaluación (antes, durante o después de la abstinencia).

Al margen de la influencia de algunos de los factores mencionados, resultan significativas las diferencias observadas en función del instrumento de evaluación utilizado. En el caso de los autoinformes, los estudios llevados a cabo con el MCMI-II (Millon, 1997) muestran unas tasas de comorbilidad del alcoholismo con los trastornos de personalidad que oscilan entre el $64 \%$ (Fernández-Montalvo et al., 2002) y el $96,7 \%$ (Echeburúa, Bravo de Medina y Aizpiri, 2005). Sin embargo, los estudios que evalúan los trastornos de personalidad con entrevistas diagnósticas — principalmente, el Structured Interview for DSM-III Personality Disorders (SDIP) (Pfohl, Stangl y Zimmerman, 1983), el SCID-II (Spitzer et al., 1989) y el IPDE (Loranger, 1995) - muestran tasas de comorbilidad sensiblemente más bajas, que oscilan por término medio entre el $30 \%$ y el $50 \%$ de los casos. Un resumen de los resultados obtenidos en los diferentes estudios, en función del instrumento de evaluación utilizado, se presenta en la Tabla 1.

Esta falta de concordancia se observa más claramente en los escasos estudios que comparan los autoinfomes y las entrevistas de evaluación en una misma muestra clínica. Así, en el estudio de Echeburúa et al. (2005) la tasa de prevalencia de trastornos de personalidad en una muestra de 30 alcohólicos era del $40 \%$ con el IPDE y del $96,7 \%$ con el MCMI-II, con diferencias estadísticamente significativas entre ambos instrumentos.

Algo similar ocurre en el estudio de Fernández-Montalvo, Landa, López-Goñi y Lorea (2006). La prevalencia de trastornos de personalidad en una muestra de 50 alcohólicos era 
Tabla 1. Prevalencia de los trastornos de personalidad en el alcoholismo

\begin{tabular}{lcc}
\hline \multicolumn{1}{c}{ Autores } & Tasa & Entrevista \\
\hline Zimmerman y Coryell (1989) & $43,4 \%$ & SDIP \\
De Jong et al. (1993) & $78 \%$ & SDIP \\
Nurnberg et al. (1993) & $64 \%$ & SCID \\
Pettinati et al. (1999) & $35,6 \%$ & SCID \\
Powell y Peveler (1996) & $24 \%$ & SCID-II \\
Morgenstern et al. (1997) & $57,9 \%$ & SCID-Il \\
Driessen et al. (1998) & $34 \%$ & IPDE \\
Verheul et al. (1998b) & $31 \%$ & IPDE, CIDI \\
Echeburúa et al. (2005) & $40 \%$ & IPDE \\
Landa et al. (2005) & $22 \%$ & IPDE \\
Grant et al. (2004) & $28,6 \%$ & Criterios DSM-IV \\
\hline & Autores & Cuestionario \\
\hline Verheul et al. (1998a) & $54 \%$ & PDQR \\
Fernández-Montalvo et al. (2002) & $64 \%$ & MCMI-II \\
Pedrero et al. (2003) & $76 \%$ & MCMI-II \\
Echeburúa et al. (2005) & $96,7 \%$ & MCMI-II \\
Fernández-Montalvo et al. (2006) & $52 \%$ & MCMI-II \\
\hline
\end{tabular}

Nota: SDIP: Structured Interview for DSM-III Personality Disorders; SCID: Structured Clinical Interview for DSM-III; IPDE: International Personality Disorders Examination; CIDI: Composite International Diagnostic Interview; PDQ-R: Personality Disorders Questionnaire-Revised; MCMI-II: Millon Clinical Multiaxial Inventory-II.

del $22 \%$ con el IPDE. Sin embargo, cuando se utilizó el MCMI-II con la misma muestra clínica, la tasa aumentaba al $52 \%$ de los casos, también con diferencias significativas entre ambos instrumentos.

\section{JUEGO PATOLÓGICO}

Por lo que se refiere al juego patológico, son escasos los estudios llevados a cabo sobre la pre- valencia de los trastornos de personalidad. Sin embargo, también se observan aquí importantes discrepancias entre las distintas investigaciones, con tasas de comorbilidad que oscilan entre el $25 \%$ y el $93 \%$ (Tabla 2). Además, tampoco hay un acuerdo en cuanto al tipo de trastorno de personalidad más frecuentemente diagnosticado en los ludópatas, si bien destacan ligeramente el trastorno antisocial y el trastorno límite (Fernández-Montalvo y Echeburúa, 2001).

Tabla 2. Prevalencia de los trastornos de personalidad en el juego patológico

\begin{tabular}{llc}
\hline \multicolumn{1}{c}{ Autores } & Tasa & Entrevista \\
\hline Specker et al. (1996) & $25 \%$ & SCID-II \\
Fernández-Montalvo y Echeburúa (2004) & $32 \%$ & IPDE \\
\hline \multicolumn{1}{c}{ Autores } & Tasa & Cuestionario \\
\hline Lesieur y Blume (1990) & $71 \%$ & $i ?$ \\
Blaszczynski y Steel (1998) & $93 \%$ & PDQ-R \\
Black y Moyer (1998) & $87 \%$ & PDQ-IV \\
Fernández-Montalvo y Echeburúa (2006) & $40 \%$ & MCMI-II \\
\hline
\end{tabular}

Nota: ¿? El instrumento de evaluación utilizado no figura especificado en el estudio. SCID: Structured Clinical lnterview for DSMIII; IPDE: International Personality Disorders Examination; PDQ-R: Personality Disorders Questionnaire-Revised; PDQ-IV: Personality Disorders Questionnaire-4 ${ }^{\text {th }}$ edition; MCMI-II: Millon Clinical Multiaxial Inventory-II. 
Las tasas tan elevadas de trastornos de personalidad entre los ludópatas (superiores al $70 \%$ en la mayor parte de los estudios, e incluso al $90 \%$ en alguno de ellos) resultan desmesuradas. Es más, el número medio de trastornos de personalidad encontrados en cada jugador (más de 4 por paciente como media) parece, cuando menos, excesivo. Todo ello refleja, probablemente, la imprecisión de los instrumentos de medida, así como la insuficiencia de los criterios diagnósticos utilizados (Fernández-Montalvo y Echeburúa, 2001).

En concreto, los estudios realizados con cuestionarios muestran una prevalencia de trastornos de personalidad que oscila, como media, en torno al 70\% (Black y Moyer, 1998; Blaszczynski y Steel, 1998; Fernández-Montalvo y Echeburúa, 2006). Estas tasas son sensiblemente más bajas cuando las investigaciones se llevan a cabo con entrevistas clínicas, con una tasa media de alrededor del $28 \%$ (Fernández-Montalvo y Echeburúa, 2004; Specker, Carlson, Edmonton, Johnson y Marcotte, 1996).

Asimismo, al igual que se ha señalado en el caso del alcoholismo, cuando se comparan los resultados obtenidos con el IPDE y con el MCMI-II en una misma muestra clínica, se observan diferencias importantes entre ambos instrumentos. Así, en el único estudio comparativo hasta la fecha (Echeburúa y FernándezMontalvo, 2006) la tasa de prevalencia de los trastornos de personalidad en una muestra de 50 ludópatas era del $32 \%$ con el IPDE y del $40 \%$ con el MCMI-II, con diferencias significativas entre ambos instrumentos.

\section{ADICCIÓN A LA COCAÍNA}

El consumo de cocaína ha experimentado en nuestro país un notable aumento en los últimos años. De hecho, por lo que se refiere a las drogas ilegales, la adicción a estimulantes representa un grave problema con el que se encuentran los programas de tratamiento de las drogodependencias (Bobes, Sáez, González y Bascarán, 2001; Caballero, 2000; Observatorio Europeo de las Drogas y las Toxicomanias,
2004; Observatorio Español sobre Drogas, 2003). Ello ha provocado un aumento - aunque todavía escaso- de los programas específicos de intervención para esta problemática.

De forma simultánea al aumento del consumo de cocaína, se ha producido un interés creciente por el estudio de los trastornos duales en el ámbito de las conductas adictivas (Tenorio y Marcos, 2000). El esfuerzo por mejorar las tasas de éxito de los programas de intervención con pacientes adictos ha generado un claro interés por el estudio de las variables que limitan o, cuando menos, modulan el alcance de dichos programas. En este sentido ha cobrado una especial importancia el análisis de las dimensiones de personalidad más frecuentes en este tipo de población (Sáiz, González, Paredes, Martínez y Delgado, 2001) y, más recientemente, el estudio de los trastornos concretos de la personalidad implicados (Fernández-Montalvo, Lorea, López-Goñi y Landa, 2003).

Las tasas de comorbilidad de la adicción a la cocaína con los trastornos de personalidad oscilan entre el $45 \%$ y el $70 \%$ (Tabla 3 ), con un cierto predominio del trastorno límite de la personalidad. En este caso también se observa una amplia variabilidad entre los distintos estudios, aunque no se puede atribuir exclusivamente al tipo de instrumento de evaluación utilizado porque más de la mitad de los estudios recurren al mismo instrumento (el SCID-II). Probablemente el hecho de contar aún con un reducido número de estudios dificulta la obtención de conclusiones más definitivas.

\section{TRASTORNOS DE LA CONDUCTA ALIMENTARIA}

Los estudios sobre comorbilidad entre los trastornos de la conducta alimentaria y los trastornos de personalidad son relativamente recientes. Los resultados encontrados hasta la fecha muestran una tasa de comorbilidad muy elevada, que oscila entre el $20 \%$ y el $80 \%$ de los casos (Tabla 4). También es muy variable el tipo de trastornos implicados. En el caso de la anorexia restrictiva destacan, por su frecuencia, 
Tabla 3. Trastornos de personalidad en la adicción a la cocaína

\begin{tabular}{lcc}
\hline \multicolumn{1}{c}{ Autores } & Tasa & Entrevista \\
\hline Kranzler et al. (1994) & $70 \%$ & SCID-II \\
Barber et al. (1996) & $47 \%$ & SCID-II \\
Sonne y Brady (1998) & $68,1 \%$ & SCID-II \\
Sanz y Larrazábal (2002) & $64 \%$ & IPDE \\
\hline \multicolumn{1}{c}{ Autores } & Tasa & Cuestionario \\
\hline Fernández-Montalvo et al. (2003) & $45,7 \%$ & MCMI-II \\
\hline
\end{tabular}

Nota: SCID: Structured Clinical Interview for DSM-III; IPDE: International Personality Disorders Examination; MCMI-II: Millon Clinical Multiaxial Inventory-II.

los trastornos del grupo C, sobre todo la personalidad obsesiva y evitadora. En cambio, en la anorexia purgativa destaca la personalidad límite, lo que también ocurre en la bulimia nerviosa (Echeburúa y Marañón, 2001).

No obstante, al igual que en los ejemplos anteriores, se observan también discrepancias significativas entre las distintas investigaciones en función de los instrumentos de evaluación utilizados. En concreto, los estudios que utilizan autoinformes muestran una tasa de comorbilidad que oscila entre el $72 \%$ y el $100 \%$ (Norman, Blais y Herzog, 1993; Kennedy, et al., 1995; Del Río, Torres y Borda, 2002; Echeburúa, Marañón y Grijalvo, 2002). Sin embargo, estos porcentajes tan eleva- dos de comorbilidad descienden de forma relevante cuando se recurre a entrevistas estructuradas. En estos casos las tasas de comorbilidad oscilan entre el $26 \%$ y el $75 \%$ (Gartner, Marcus, Halmi y Loranger, 1989; Kennedy et al., 1995; Marañón, Echeburía y Grijalvo, 2004; Matsunaga, Kiriike, Nagata y Yamagami, 1998; DíazMarsa, Carrasco y Saiz, 2000; Matsunaga et al., 2000).

Nuevamente estas discrepancias se observan más claramente en aquellos estudios que comparan los autoinfomes y las entrevistas de evaluación con una misma muestra clínica. Así, en el estudio de Marañón, Grijalbo y Echeburúa (2006) la tasa de trastornos de personalidad en

Tabla 4. Prevalencia de los trastornos de personalidad en los trastornos de la alimentación

\begin{tabular}{lcc}
\hline \multicolumn{1}{c}{ Autores } & Tasa & Entrevista \\
\hline Wonderlich et al. (1990) & $72 \%$ & SCID-I \\
Matsunaga et al. (1998) & $51 \%$ & SCID-II \\
Diaz-Marsa et al. (2000) & $61,8 \%$ & SCID-II \\
Godt (2002) & $33 \%$ & SCID-II \\
Gartner et al. (1989) & $57 \%$ & PDE \\
Grilo et al. (1996) & $84 \%$ & PDE \\
Karwautz et al. (2002) & $54 \%$ & IPDE \\
Marañón et al.(2004) & $51,5 \%$ & IPDE \\
Marañón et al. (2006) & $54,8 \%$ & IPDE \\
\hline \multicolumn{1}{c}{ Autores } & Tasa & Cuestionario \\
\hline Inceoglu et al. (2000) & $49,2 \%$ & PDQ-R \\
Kennedy et al. (1990) & $93 \%$ & MCMI \\
Norman et al. (1993) & $84 \%$ & MCMI \\
Del Río et al. (2002) & $81 \%$ & MCMI-II \\
Marañón et al. (2006) & $77,4 \%$ & MCMI-II \\
\hline
\end{tabular}

Nota: SCID: Structured Clinical Interview for DSM-III; PDE: Personality Disorders Examination; IPDE: International Personality Disorders Examination; PDQ-R: Personality Disorders Questionnaire-Revised; MCMI-II: Millon Clinical Multiaxial Imventory-II. 
una muestra de 84 mujeres afectadas por distintos trastornos de la conducta alimentaria era del $54,8 \%$ con el IPDE y del $77,4 \%$ con el MCMIII, con diferencias significativas entre ambos instrumentos.

\section{CONCLUSIONES}

El avance experimentado en los últimos años en el conocimiento de los trastornos de personalidad ha provocado un aumento importante de estudios sobre su comorbilidad con los trastornos mentales del Eje I. Sin embargo, los resultados encontrados hasta la fecha muestran muchas discrepancias entre los distintos estudios, con tasas muy amplias y variadas de prevalencia de los trastornos de personalidad. Al margen de algunos factores que pueden influir en la amplia variabilidad encontrada -el tipo de muestra utilizada o el momento específico de evaluación, por ejemplo--, resulta curioso observar los resultados tan dispares encontrados según sean los instrumentos de evaluación a los que se ha recurrido.

En este artículo se ha llevado a cabo una revisión, sin pretensiones de exhaustividad, de las discrepancias halladas en los estudios de comorbilidad. Como se ha puesto de manifiesto reiteradamente, las investigaciones que utilizan cuestionarios tienden a encontrar tasas significativamente más altas de trastornos de personalidad que las que recurren a entrevistas estructuradas. Se puede, por ello, concluir que los autoinformes presentan una tendencia a sobrediagnosticar trastornos de personalidad. Las entrevistas clínicas, en cambio, son más estrictas y conservadoras, por lo que las tasas de prevalencia son más bajas, incluso con diferencias significativas cuando se utilizan ambos tipos de instrumentos con una misma muestra clínica. Las ventajas aparentes de los cuestionarios autoadministrados en el ámbito clínico -su mayor comodidad, el ahorro de costes y de tiempo, etcétera- comportan un detrimento en la calidad de la evaluación y los hace, por tanto, poco fiables para, al menos, el diagnóstico de los trastornos del Eje II.

Probablemente la causa de este hecho no se deba en exclusiva a los instrumentos de evalua- ción utilizados. Los trastornos de personalidad constituyen hoy por hoy una categoría diagnóstica de gran imprecisión conceptual (Livesley, 2001; Livesley y Jang, 2000). Como consecuencia, el diagnóstico de los trastornos de personalidad en la práctica clínica tiende a ser poco fiable, en parte por la definición poco precisa de estos trastornos y en parte por la inexistencia de instrumentos de medida adecuados (Jacobsberg, Goldsmith, Widiger y Frances, 1991; Valdés, 2000).

Los trastornos de personalidad se caracterizan por la presencia de muchos síntomas egosintónicos y socialmente indeseables, de los que el sujeto no es consciente o que tiende a ocultar. Por ello, su criterio sobre su propia conducta no puede constituirse en el único punto de referencia. No es muy adecuado, por ejemplo, preguntarle a un paciente con una personalidad antisocial si ha mentido repetidamente, si le importa o no la verdad o si carece de remordimientos. Asimismo determinados cuadros clínicos presentan síntomas que también forman parte de algunos trastornos de personalidad. Un ludópata, por ejemplo, puede contestar afirmativamente a todos los ítems de un cuestionario que pregunten acerca de los robos y de las mentiras, que son dos características habituales del juego patológico. Sin embargo, ello no significa necesariamente que presente un trastorno antisocial de la personalidad, aunque la mera corrección del autoinforme así lo indique. Parece, por tanto, desaconsejable utilizar cuestionarios para evaluar trastornos de personalidad en pacientes con una clara ausencia de conciencia de enfermedad y/o que presentan síntomas socialmente indeseables (Westen y Shedler, 1999a, 1999b).

Por otra parte, el diagnóstico de un trastorno de personalidad precisa ahondar más en las características específicas del trastorno y valorar en qué medida obedece a una personalidad alterada o a un cuadro clínico concreto. La evaluación de los trastornos de personalidad requiere, por definición, una evaluación longitudinal, es decir, de toda la biografia de la persona. Ello supone una gran diferencia -y una dificultad adicional- respecto a la evaluación de 
los trastornos mentales del Eje I, que suele ser más transversal y toma en consideración prioritariamente los síntomas presentes en la actualidad (Valdés, 2000, 2002).

Las fuentes de información actualmente disponibles son: a) las entrevistas y el juicio del clínico; b) los cuestionarios autoadministrados; y c) las informaciones complementarias de los familiares o personas que conviven con el paciente. Respecto a esta última fuente, el papel de la misma puede no ser importante en las alteraciones del Eje I (porque el síndrome suele ser inmediatamente observable), pero sí en los trastornos de personalidad (por el carácter histórico de dichos trastornos). Si bien la validez de cada una de estas fuentes está aún por establecer (Widiger y Frances, 1987), la utilización conjunta de todas ellas, así como la observación a lo largo del tiempo, parecen potenciar la validez del diagnóstico.

En cualquier caso, el futuro en la evaluación de los trastornos de personalidad pasa por el desarrollo de entrevistas clínicas más ajustadas y menos farragosas que las disponibles en la actualidad, limitadas en gran medida a los estudios de investigación. De poco sirve conocer las imprecisiones de los cuestionarios si la alternativa a la evaluación de los trastornos de personalidad es la utilización de entrevistas clinicas más o menos estructuradas, que hoy por hoy son excesivamente largas $\mathrm{y}$, por tanto, de difícil aplicación en un contexto clínico cotidiano.

\section{REFERENCIAS}

Baca-Baldomero, E. (1999). Prefacio. En M. Bernardo y M. Roca (Eds.). Trastornos de personalidad. Evaluación y tratamiento. Barcelona: Masson.

Barber, J.B., Frank, A., Weiss, R.D., Blaine, J., Siqueland, L., Moras, K., et al. (1996). Prevalence and correlates of personality disorder diagnoses among cocaine dependent outpatients. Journal of Personality Disorders, 10, 297-311.

Bernardo, M. y Roca, M. (1999). Introducción. En M. Bernardo y M. Roca (Eds.), Trastornos de personalidad. Evaluación y tratamiento. Barcelona: Masson.
Black, D.W. \& Moyer, T. (1998). Clinical features and psychiatric comorbidity of subjets with pathological gambling behavior. Psychiatric Services, 49, 1434-1439.

Blaszczynski, A. \& Steel, Z. (1998). Personality disorders among pathological gamblers. Journal of Gambling Studies, 14, 51-71.

Bobes, J., Sáiz, P.A., González, M.P. y Bascarán, M.T. (2001). Epidemiología del uso/abuso de cocaína. Adicciones, 13, 23-36.

Caballero, L. (2000). Adicción a la cocaina: de la neurobiología a la clínica humana. Jano, 2, 5-9.

Caballo, V.E. (2004). Conceptos actuales sobre los trastornos de la personalidad. En V.E. Caballo (Ed.), Manual de trastornos de la personalidad. Madrid: Síntesis.

Calcedo, A. (2000). Evaluación forense. En J. Bobes, M. Bousoño, A. Calcedo y M.P. González (Eds.), Trastorno de estrés postraumático. Barcelona: Masson.

Cangas, A.J. y Olivencia, J.J. (2001). Relación de los trastornos de la personalidad con el seguimiento de instrucciones terapéuticas y las recaídas en sujetos drogodependientes. Psicologia Conductual, 9, 541-549.

Cañas de Paz, F. (1998). Instrumentos de evaluación. En M. Bernardo y M. Roca (Eds.), Trastornos de la personalidad. Evaluación y tratamiento. Barcelona: Masson.

De Jong, C.A., Van den Brink, W., Harteveld, F.M. y Van der Wielen, E.G. (1993). Personality disorders in alcoholics and drug addicts. Comprehensive Psychiatry, 34, 87-94

De la Gándara, J.J. y Medina, M.A. (1998). Comorbilidad. En M. Bernardo y M. Roca (Eds.), Trastornos de la personalidad. Evaluación y tratamiento. Barcelona: Masson.

Del Río, C., Torres, I. y Borda, M. (2002). Comorbilidad entre bulimia nerviosa purgativa y trastornos de la personalidad según el Inventario Clínico Multiaxial de Millon (MCMI-II). International Journal of Clinical and Health Psychology, 2, 425-438.

Díaz-Marsa, M., Carrasco, J.L. y Saiz, J. (2000). A study of temperament and personality in anorexia and bulimia nervosa. Journal of Personality Disorders, 14, 352-359.

Driessen, M., Veltrup, C., Wetterling, T., John, U. y Dilling, H. (1998). Axis I and axis II comorbidity in alcohol dependence and the two types of alcoholism. Alcoholism: Clinical and Experimental Research, 22, 77-86.

Dutton, D. (2003). MCMI results for batterers: A response to Gondolf. Journal of Family Violence, $18,253-255$. 
Echeburúa, E., Amor, P.J. y Corral, P. (2003). Autoinformes y entrevistas en el ámbito de la psicología clínica forense: Limitaciones y nuevas perspectivas. Análisis y Modificación de Conducta, 29, 503-522.

Echeburúa, E., Bravo de Medina, R. y Aizpiri, J. (2005). Alcoholism and personality disorders: An exploratory study. Alcohol and Alcoholism, 40, 323-326.

Echeburúa, E. y Fernández-Montalvo, J. (2006, en prensa). Are there more personality disorders in treatment-seeking pathological gamblers than in other kind of patients? A comparative study with the IPDE and the MCMI. Journal of Personality Assessment.

Echeburúa, E. y Marañón, I. (2001). Comorbilidad de las alteraciones de la conducta alimentaria con los trastornos de personalidad. Psicología Conductual, 7, 95-101.

Echeburúa, E., Marañón, I. y Grijalvo, J. (2002). Trastornos de personalidad en pacientes aquejadas de anorexia y bulimia nerviosa: Un estudiopiloto. Revista de Psicopatología y Psicología Clínica, 7, 95-101.

Fernández-Montalvo, J. y Echeburúa, E. (2001). Trastornos de personalidad y juego patológico: una revisión crítica. Psicologia Conductual, 9, 527-539.

Fernández-Montalvo, J. y Echeburúa, E. (2004). Pathological gambling and personality disorders: An exploratory study with the IPDE. Journal of Personality Disorders, 18, 500-505.

Fernández-Montalvo, J. y Echeburúa, E. (2006, en prensa). Juego patológico y trastornos de personalidad: un estudio-piloto con el MCMI-II. Psicothema.

Fernández-Montalvo, J. y Landa, N. (2003). Comorbilidad del alcoholismo con los trastornos de personalidad. Clinica y Salud, 14, 27-41.

Fernández-Montalvo, J., Landa, N. y López-Goñi, J.J. (2005). Prevalencia del juego patológico en el alcoholismo: un estudio exploratorio. Revista de Psicopatologia y Psicología Clínica, 10, 125133.

Fernández-Montalvo, J., Landa, N., López-Goñi, J.J. y Lorea, I. (2006, en prensa). Personality disorders in alcoholics: A comparative pilot study between the IPDE and the MCMI-II. Addictive Behaviors.

Fernández-Montalvo, J., Landa, N., López-Goñi, J.J., Lorea, I. y Zarzuela, A. (2002). Trastornos de personalidad en alcohólicos: Un estudio descriptivo. Revista de Psicopatología y Psicologia Clinica, 7, 217-225.

Fernández-Montalvo, J., López-Goñi, J.J., Landa, N., Illescas, C., Lorea, I. y Zarzuela, A. (2004). Tras- tornos de personalidad y abandonos terapéuticos en pacientes adictos: resultados en una comunidad terapéutica. International Journal of Clinical and Health Psychology, 4, 271-283.

Fernández-Montalvo, J., Lorea, I., López-Goñi, J.J. y Landa, N. (2003). Trastornos de personalidad en adictos a la cocaína: Un estudio-piloto. Análisis y Modificación de Conducta, 29, 79-98.

Gartner, A., Marcus, R., Halmi, K. y Loranger, A. (1989). DSM-III personality disorders in patients with eating disorders. American Journal of Psychiatry, 146, 1585-1591.

Girolamo, G. y Reich, J.H. (1993). Epidemiology of mental disorders and psychosocial problems: personality disorders. Ginebra. World Health Organization.

Godt, K. (2002). Personality and eating disorders: the prevalence of personality disorders in 176 female outpatients with eating disorders. European Eating Disorders Review, 10, 102-109.

Gondolf, E.W. (2003). MCMI results for batterers: Gondolf replies to Dutton's response. Journal of Family Violence, 18, 387-389.

Grant, B.F., Stinson, F.S., Dawson, D.A., Chou, S.P., Ruan, W.J. y Pickering, R.P. (2004). Co-occurrence of 12-month alcohol and drug use disorders and personality disorders in the United States: results from the National Epidemiologic Survey on Alcohol and Related Conditions. Archives of General Psychiatry, 61, 361-368.

Grilo, C.M., Levy, K.N., Becker, D.F., Edell, W.S. y McGlashan, T.H. (1996). Comorbidity of DSMIII-R Axis I and II disorders among female inpatients with eating disorders. Psychiatric Services, 47, 426-429.

Hyler, S.E., Reider, R.C., Williams, J.B.W., Spitzer, R.L., Lyons, M. y Hendler, J. (1989). A comparison of clinical and self report diagnoses of DSMIII personality disorders in 552 patients. Comprehensive Psychiatry, 30, 170-178.

Inceoglu, I., Franzen, U., Backmund, H. y Gerlinghoff, M. (2000). Personality disorders in patients in a day-treatment programme for eating disorders. European Eating Disorders Review, 8, 67-72.

Jacobsberg, L.B., Goldsmith, S., Widiger, T. y Frances, A.J. (1991). Evaluación de los trastornos de personalidad del DSMIII. En S. Wetzler (Ed.), Medición de las enfermedades mentales. Evaluación psicométrica para los clínicos. Barcelona: Ancora.

Karwautz, A., Rabe-Hesketh, S., Collier, D.A. y Treasure, J.L. (2002). Premorbid psychiatric morbidity, comorbidity and personality in patients with anorexia nervosa compared to their healthy sisters. European Eating Disorders Review, 10, 255-270. 
Kennedy, S.H., Katz, R., Rockert, W., Mendlowitz, S., Ralevski, E. y Clewes, J. (1995). Assessment of personality disorders in anorexia nervosa and bulimia nervosa: a comparison of self report and structures interview methods. The Journal of Nervous and Mental Disease, 183, 358-364.

Kennedy, S.H., McVey, G. y Katz, R. (1990). Personality disorders in anorexia nervosa and bulimia nervosa. Journal of Psychiatric Research, 24, 259-269.

Kranzler, H.R., Satel, S. y Apter, A. (1994). Personality disorders and associated features in cocaine-dependent inpatients. Comprehensive Psychiatry, 35, 335-340.

Landa, N., Fernández-Montalvo, J., López-Goñi, J.J. y Lorea, I. (2005). Trastornos de personalidad en alcohólicos: un estudio con el IPDE. Psicología Conductual, 13, 275-288.

Lesieur, H.R. \& Blume, S.B. (1990). Characteristics of pathological gamblers identified among patients on a psychiatric admission service. Hospital and Community Psychiatry, 41, 1009-1012.

Livesley, W.J. (2001). Conceptual and taxonomic issues. En W.J. Livesley (Ed.), Handbook of personality disorders. Nueva York: Guilford.

Livesley, W.J. y Jang, K.L. (2000). Toward an empirically bases classification of personality disorders. Journal of Personality Disorders, 14, 137-147.

Loranger, A.W. (1995). Personality Disorder Examination (IPDE) Manual. Yonkers. D V Communication.

Marañón, I., Echeburúa, E. y Grijalvo, J. (2004). Prevalence of personality disorders in patients with eating disorders: a pilot-study using the IPDE. European Eating Disorders Review, 12, 217-222.

Marañón, I., Grijalvo, J. y Echeburúa, E. (2006, en prensa). Do the IPDE and the MCMI assess the same personality disorders in patients with eating disorders?. Journal of Psychopathology and Behavioral Assessment.

Matsunaga, H., Kaye, W.H., McConaha, C., Plotnicov, K., Pollice, C. y Rao, R. (2000). Personality disorders among subjects recovered from eating disorders. International Journal of Eating Disorders, 27, 353-357.

Matsunaga, H., Kiriike, N., Nagata, T. y Yamagami, S. (1998). Personality disorders in patients with eating disorders in Japan. International Journal of Eating Disorders, 23, 399-408.

Millon, T. (1997). Millon Clinical Multiaxial Inventory-II (MCMI-II). Minneapolis: National Computer Systems.

Morgenstern, J., Langenbucher, J., Labouvie, E. y Miller, K. (1997). The comorbidity of alcoholism and personality disorders in a clinical population: prevalence rates and relation to alcohol typology variables. Journal of Abnormal Psychology, 106, 74-84.

Nace, E.P., Davis, C.W. y Gaspari, J.P. (1991). Axis II comorbidity in substance abusers. American Journal of Psychiatry, 148, 118-120.

Norman, D., Blais, M.A. y Herzog, D. (1993). Personality characteristics of eating-disordered patients as identified by the Millon Clinical Multiaxial Inventory. Journal of Personality Disorders, 7, 1-9.

Nurnberg, H.G., Rifkin, A. y Doddi, S. (1993). A systematic assessment of the comorbidity of DSM III R personality disorder in alcoholic outpatients. Comprehensive Psychiatry, 34, 447-454.

Observatorio Español sobre Drogas (2003). Informe $N .^{\circ}$ 6. Delegación del Gobierno para el Plan Nacional sobre Drogas. Madrid: Ministerio del Interior.

Observatorio Europeo de las Drogas y las Toxicomanías (2004). Informe Anual 2004. El problema de la drogodependencia en la Unión Europea y en Noruega. Luxemburgo: Oficina de Publicaciones Oficiales de las Comunidades Europeas.

Pedrero, J., Puerta, C., Lagares, A. y Sáez, A. (2003). Prevalencia e intensidad de trastornos de personalidad en adictos a sustancias en tratamiento en un centro de atención a las drogodependencias. Trastornos Adictivos, 5, 241-255.

Pedrero, J. y Segura, I. (2003). Los trastornos de personalidad en drogodependientes y su relación con la dificultad de manejo clínico. Trastornos Adictivos, 3, 229-240

Perry, J.C. (1992). Problems and considerations in the valid assessment of personality disorders. American Journal of Psychiatry, 149, 1645-1653.

Pettinati, H., Pierce, J., Belden, P. y Meyers, K. (1999). The relationship of axis II personality disorders to other known predictors of addiction treatment outcome. The American Journal on Addictions, 8, 136-147.

Pfohl, B., Stangl, D. y Zimmerman; M. (1983). The Structured Interview for DSM III Personality Disorders. 2nd ed. Iowa City, IA: University of Iowa Hospitals and Clinics.

Poldrugo F. y Forti, B. (1988). Personality disorders and alcoholism treatment outcomes. Drug and Alcohol Dependence, 21, 171-176.

Powell, G. y Peveler, R. (1996). Nature and prevalence of personality disorders amongst patients receiving treatment for alcohol dependence. Journal of Mental Health, 5, 305-314.

Sáiz, P.A., González, M.P., Paredes, B., Martínez, S. y Delgado, J.M. (2001). Personalidad y uso-abuso de cocaína. Adicciones, 13, 47-59.

Samino, F.J. y Lorenzo, M.J. (2003). Evaluación de los trastornos de personalidad. Instrumentos diag- 
nósticos. En V. Rubio y A. Pérez Urdaniz (Eds.). Trastornos de la personalidad. Madrid: Elsevier.

Sanz, J. y Larrazábal, A. (2002). Comorbilidad de dependencia de cocaína y trastornos de personalidad. Interpsiquis. Extraído el 31 de enero de 2006 de http://www.psiquiatria.com/articulos/adicciones/5592.

Sonne, S.C. y Brady, K.T. (1998). Diagnosis of personality disorders in cocaine-dependent individuals. The American Journal on Addictions, 7, 1-6.

Specker, S.M., Carlson, G.A., Edmonson, K.M., Johnson, P.E. y Marcotte, M. (1996). Psychopathology in pathological gamblers seeking treatment. Journal of Gambling Studies, 12, 67-82.

Spitzer, R.L., Williams, J.B. y Sibbon, M. (1989). Structured Clinical Interview for DSM-III-R, Axis II. SCID-II. Rev. New York, N.Y. Biometrics Research Department. New York State Psychiatric Institute.

Tenorio, J. y Marcos, J.A. (2000). Trastornos duales: tratamiento y coordinación. Papeles del Psicólogo, 77, 58-63.

Valdés, M. (2000). Trastornos de la personalidad. En A. Bulbena, G.E. Berrios y P. Fernández de Larrinoa (Eds.), Medición clínica en psiquiatría y psicologia. Barcelona: Masson.

Valdés, M. (2002). Trastornos de personalidad. En J. Vallejo (Ed.), Introducción a la psicopatología y la psiquiatria. Barcelona: Masson.

Verheul, R., Van den Brink W. y Hartgers, C. (1995). Prevalence of personality disorders among alco- holics and drug addicts: An overview. European Addiction Research, 1, 166-177.

Verheul, R., Van den Brink, W. y Hartgers, C. (1998a). Personality disorders predict relapse in alcoholic patients. Addictive Behaviors, 23, 869882.

Verheul, R., Hartgers, C., Van den Brink, W. y Koeter, M.W. (1998b). The effect of sampling, diagnostic criteria and assessment procedures on the observed prevalence of DSM-III-R personality disorders among treated alcoholics. Journal of Studies on Alcohol, 59, 227-236.

Westen, D. y Shedler, J. (1999a). Revising and assessing Axis II. Part I: Developing a clinically and empirically valid assessment method. American Journal of Psychiatry, 156, 258-272.

Westen, D. y Shedler, J. (1999b). Revising and assessing Axis II. Part II: Toward an empirically based and clinically useful classification of personality disorders. American Journal of Psychiatry, 156, 273-285.

Widiger, T.A. y Frances, A. (1987). Interviews and inventories for the measurement of personality disorders. Clinical Psychology Review, 7, 49-75.

Wonderlich, S.A., Swift, W.J., Slotnick, H.B. y Goodman, S. (1990). DSM-III-R personality disorders in eating disorders subtypes. International Journal of Eating Disorders, 9, 607-616.

Zimmerman, M. y Coryell, W. (1989). DSM III personality disorder diagnosis in a nonpatient sample. Demographic correlates and comorbidity. Archives of General Psychiatry, 46, 682-689. 